\title{
Belphégor
}

Littérature populaire et culture médiatique

10-3 | 2011

Peter Pan

\section{Réenchanter le monde : Barrie lu par Rackham}

\section{François Fièvre}

\section{OpenEdition}

\section{Journals}

Édition électronique

URL : http://journals.openedition.org/belphegor/395

DOI : 10.4000/belphegor.395

ISSN : 1499-7185

Éditeur

LPCM

Édition imprimée

Date de publication : 10 janvier 2011

Référence électronique

François Fièvre, « Réenchanter le monde : Barrie lu par Rackham », Belphégor [En ligne], 10-3 | 2011, mis en ligne le 10 janvier 2013, consulté le 30 avril 2019. URL : http://journals.openedition.org/ belphegor/395 ; DOI : 10.4000/belphegor.395

Ce document a été généré automatiquement le 30 avril 2019.

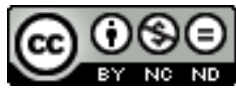

Belphégor est mis à disposition selon les termes de la Licence Creative Commons Attribution - Pas d'Utilisation Commerciale - Pas de Modification 4.0 International. 


\title{
Réenchanter le monde : Barrie lu par Rackham
}

\author{
François Fièvre
}

1 La collaboration d'Arthur Rackham à l'œuvre de Barrie est bien connue de ceux qui s'intéressent à la genèse du personnage de Peter Pan. Ses illustrations sont parfois utilisées pour orner les couvertures des éditions, anglaises comme françaises, contenant les différents textes que Barrie a consacrés à son si célèbre héros ${ }^{1}$. Paradoxe s'il en est, sachant que c'est le second épisode des aventures de Peter Pan qui a connu le succès que l'on sait, alors qu'Arthur Rackham ne s'est confronté qu'au texte du premier épisode, qui se déroule dans les jardins de Kensington et non à Neverland : un épisode peu connu du grand public, mais qui mérite d'être redécouvert tant du fait de son importance dans la genèse du personnage de Barrie que de ses qualités littéraires propres².

2 Ce qui nous intéressera ici au premier chef est néanmoins, plus que le texte de Peter Pan in Kensington Gardens, la manière dont Arthur Rackham l'a interprété en images. La problématique de l'illustration des textes littéraires s'articule très souvent autour de la question de la distance ou de la proximité que l'image entretient vis-à-vis du texte - de ses fidélités ou de ses trahisons. Variation sur le fameux ut pictura poesis d'Horace, cette question est fréquemment au centre des réflexions des artistes qui se confrontent à un imaginaire textuel, et détermine en grande partie leur légitimité en tant qu'artistes: doivent-ils imiter servilement la lettre du texte, ou peuvent-ils au contraire s'accorder la licence poétique d'interpréter celui-ci à leur manière? Arthur Rackham, considéré comme l'un des plus grands illustrateurs de son temps, répond de manière assez unilatérale à cette alternative : l'illustrateur ne doit pas se contenter de produire de belles images, mais doit savoir interpréter le texte et en donner une version personnelle... tout en restant fidèle à son esprit, ce qui suppose une certaine affinité entre écrivain et artiste. C'est selon cette problématique que nous voulons examiner la série d'illustrations d'Arthur Rackham - qui nous permettra peut-être aussi de mieux comprendre certains aspects du texte de Barrie.

3 Examiner la manière dont Rackham s'est emparé du texte de Barrie suppose une réflexion en deux temps : tout d'abord une analyse historique qui permette d'établir, du point de 
vue de l'histoire de l'édition, la manière dont Rackham s'est approprié l'auctorialité du livre publié en 1906 en y imprimant sa marque personnelle; puis une analyse d'images qui présente la manière dont Rackham a su proposer une «lecture » de Barrie qui, pour être propre à l'artiste, ne permet pas moins de mettre en lumière, à travers la notion de « réenchantement du monde », une dimension a priori secondaire du texte.

\section{Quand un auteur s'appuie « contre » un autre}

Commençons par un simple rappel concernant la genèse du personnage de Peter Pan: celui-ci apparaît pour la première fois ${ }^{3}$ dans un roman pour adultes intitulé The Little White Bird, publié en 1902, où ses aventures sont l'objet de six chapitres (chapitres XIII à XVIII) insérés à l'intérieur de la trame principale. Ce sont ces six chapitres qui feront l'objet d'une édition à part en 1906, réunis sous le titre Peter Pan in Kensington Gardens, avec les fameuses illustrations de Rackham ${ }^{4}$. Entretemps, la pièce de théâtre qui raconte le second épisode - celui qui se déroule à Neverland - a été créée le 27 décembre 1904, et plus tard, après maintes modifications, cette pièce de théâtre sera transformée en roman, publié en 1911 sous le titre Peter and Wendy.

C'est très probablement le succès considérable de la pièce de théâtre en 1904 qui a amené Barrie - ou son éditeur habituel, Hodder \& Stoughton - à considérer d'un nouvel œil le premier épisode des aventures du héros racontées dans The Little White Bird, et à envisager sa publication sous une forme séparée. D’après Derek Hudson, biographe de Rackham, l'initiative d'une édition illustrée par Arthur Rackham reviendrait néanmoins à MM. Ernest Brown et Phillips des galeries Leicester ${ }^{5}$, pour lesquels l'artiste-illustrateur avait déjà en 1904 accepté d'illustrer Rip Van Winkle de Washington Irving, les illustrations ayant ensuite été revendues à Heinemann pour être publiées sous forme de livre illustré à la fin de l'année 1905. Les galeries Leicester avaient exposé les aquarelles de Rackham pour le conte d'Irving, assorties de 39 autres compositions de l'artiste, de mars à avril 1905, ce qui leur avait permis de créer un double événement : une exposition précédant la publication d'un livre illustrée. C'est selon une formule similaire mais améliorée (l'exposition précédant cette fois-ci directement la publication) qu'a été publié le Peter Pan de 1906 : une publication, cette fois-ci chez l'éditeur de Barrie Hodder \& Stoughton, assortie d'une exposition des originaux de Rackham aux galeries Leicester dans les mois qui précèdent la publication du livre. Cette organisation est à l'avantage du galeriste et de l'artiste tout autant que de l'éditeur et de l'auteur, et nous permet de montrer que derrière la parution de l'ouvrage se profile une opération artistique tout autant qu'une opération littéraire - on verra que la suite des opérations semble même être davantage dans les mains des galeristes que des éditeurs.

MM. Brown et Phillips organisent une entrevue entre Rackham et Barrie dès juin 1905, peu après que l'exposition des illustrations de Rip Van Winkle a terminé en avril Rackham est alors invité à Leinster Corner, chez Barrie, pour discuter de l'oeuvre et du projet éditorial ${ }^{7}$. Les galeristes n'avaient visiblement pas encore conclu avec Hodder \& Stoughton de contrat très précis : dans une lettre à Rackham, ils parlent de l'illustration du Petit Oiseau blanc «ou n'importe quel autre titre»: c'est probablement lors de cet entretien de juin 1905 qu'est prise la décision de ne pas illustrer la totalité de l'ouvrage, mais uniquement les chapitres XIII à XVIII qui y fonctionnent de manière relativement indépendante, et peuvent être extraits sans grandes modifications ${ }^{8}$. Les conditions du contrat entre les galeries Leicester et Rackham sont les suivantes: Brown et Phillips 
doivent régler 5 guinées par illustration concernant les droits d'auteur, "les dessins devant être entrepris dès maintenant, et le tout devant être livré vers le 1er septembre 1906, couverture et page de titre inclus ${ }^{9}$ ", ce qui laisse plus d'une année de travail à Rackham. Une exposition doit être organisée à partir de novembre 1906, qui accompagne ainsi la sortie du livre pour les étrennes - Rackham conserve le droit de propriété sur les originaux, contrairement à ce qui s'était passé pour Rip Van Winkle l'année précédente, et leur vente se fait donc à son bénéfice.

Rackham commence alors à travailler sur le projet, se rendant régulièrement dans les jardins pour réaliser des études préparatoires. Il y travaille visiblement été comme hiver, les illustrations finales faisant alterner des paysages de toutes les saisons. On pense notamment à « Fairies are all more or less in hiding until dusk », une illustration où le sol verdoyant et les arbres encore timidement recouverts de feuilles donnent une idée des jardins au printemps, alors que «There is almost nothing that has such a keen sense of fun as a fallen leaf » ou «An afternoon when the Gardens were white with snow » ont pour leur part probablement été conçues respectivement en automne et en hiver. Walter Starkie, le neveu de Rackham, raconte beaucoup plus tard la manière dont il a accompagné son oncle dans les jardins de Kensington pour de longues promenades au cours desquelles l'artiste, entre deux croquis, lui racontait des histoires extraordinaires :

Mon nouvel oncle m'emmena faire plusieurs visites passionnantes de Londres, mais les jours les plus extraordinaires étaient ceux où [il] m'autorisait de (sic) l'accompagner à une expédition de peinture. On partait tôt par une matinée ensoleillée et mon oncle, chargé de tout son bardas de peintures, pinceaux et chevalet, me rappelait l'un des gnomes décrits par Andrew Lang dans Blue Fairy Book ; mais une fois arrivés dans les jardins de Kensington, et le peintre armé de sa palette et de son pinceau, il devenait à mes yeux un magicien qui d'un coup de sa baguette magique allait peupler mon imagination d'elfes, de gnomes et de farfadets. Il me faisait regarder fixement l'un des arbres majestueux aux troncs massifs qui nous entouraient et me parlaient [sic] des contes de Grimm qu'il avait illustrés, et des petits hommes qui soufflaient dans leurs trompes au pays des Elfes. Il me disait que sous les racines de cet arbre les petits hommes prenaient leur dîner, et barattaient le beurre qu'ils extrayaient de la sève. Il me faisait aussi remarquer les animaux étranges et les oiseaux qui peuplaient les branches de l'arbre, et la petite porte magique en bas du tronc, qui ouvrait sur le pays des Fées ${ }^{10}$.

Rackham a visiblement fini sa série d'illustrations assez tôt, dans la mesure où il convient d'un autre rendez-vous avec Barrie pour lui montrer le travail terminé, et où celui-ci dit lui répondre avec retard le 18 juin 1906. Le retard de Barrie est dû à ses soucis personnels : c'est à cette époque qu'Arthur Llewelyn Davies - le père des enfants dont l'auteur s'est en grande partie inspiré pour créer Peter Pan - voit ses jours sévèrement menacés par un cancer de la gorge.

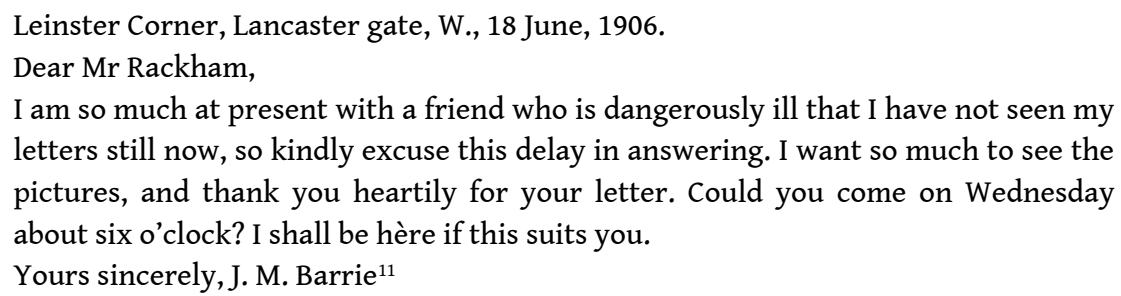

9 Peu de temps avant la sortie du livre, en octobre, Barrie se permettra également de donner des indications de dernière minute concernant la carte des jardins qui apparaît sur les premières pages de garde : 
Leinster Corner, Lancaster gate, W., 22 Oct., 1906

Dear Mr Rackham,

The Sheep shearing nowadays is done where I put the cross (behind the cottage at that point.) Fairies Basin south of Baby Walk.

I haven't seen a dummy book but H. \& S. sent me some specimen pictures which I

liked hugely.

Yours sincerely, J. M. Barrie.

On remarquera qu'à ce stade pourtant avancé l'écrivain n'a pas eu l'opportunité de voir la maquette de l'ouvrage, ce qui indique que le livre est sans doute davantage dans les mains de l'illustrateur et de l'éditeur que dans celles de l'écrivain, même si l'approbation de ce dernier est bien sûr demandée. En novembre, l'exposition aux galeries Leicester va commencer, et Barrie est naturellement invité à l'inauguration, invitation qu'il décline, étant en déplacement le jour du vernissage. Détail qui montre sans doute sinon un désintérêt, du moins un relatif désinvestissement de l'auteur pour l'entreprise : n'étant pas au centre de l'opération, Barrie ne fait pas de ce vernissage une priorité de son emploi du temps. Le livre sort ensuite pour les fêtes sous trois formes différentes : une édition de luxe, une édition britannique et une édition américaine ${ }^{12}$. L'édition de luxe est limitée à 500 exemplaires et signée par l'artiste, et non l'auteur, ce qui indique à nouveau la finalité avant tout artistique de l'ouvrage: son intérêt commercial réside avant tout dans les images, qui sont nouvelles, et non dans le texte, qui n'est pas à proprement parler inédit. Dans le marché du «gift book» édouardien, le nom de Rackham importe tout autant ici, voire plus que celui de Barrie ${ }^{13}$.

11 Finalement, ayant eu l'occasion d'aller voir l'exposition et ayant reçu son exemplaire du livre accompagné d'un dessin supplémentaire de l'artiste à son attention, Barrie fait part avec un certain retard de son enthousiasme à Rackham :

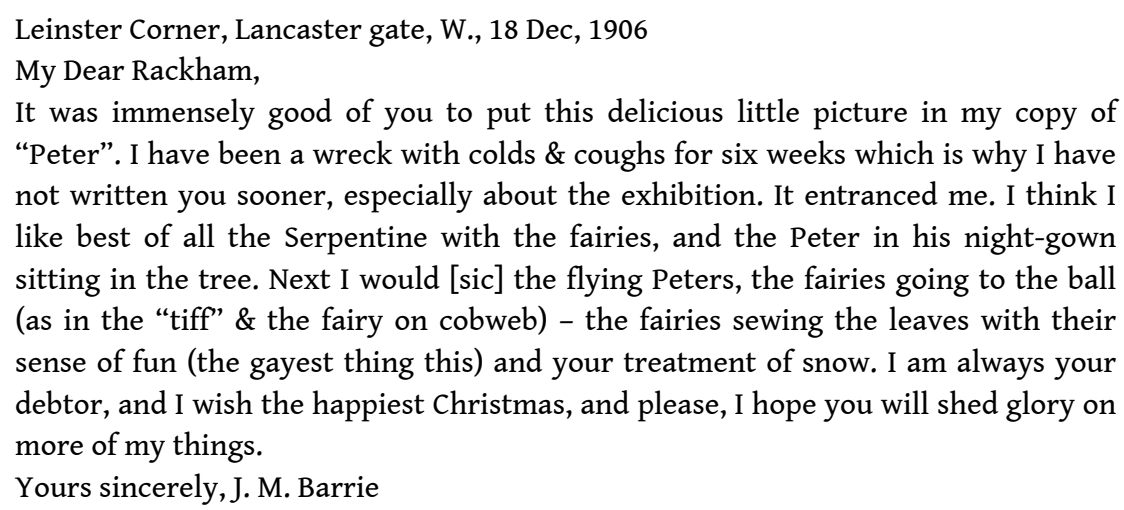

L'appel final à une collaboration ultérieure restera lettre morte: Rackham n'illustrera plus d'autre texte pour Barrie. Faut-il prendre cet espoir d'une collaboration future comme une simple politesse d'usage ? La brièveté de la lettre de remerciement de Barrie, envoyée six semaines après le début de l'exposition, qui répertorie les quelques illustrations préférées et finit par un commentaire sur « le traitement de la neige » sans formuler d'opinion générale sur le travail de Rackham, tendrait en tout cas à montrer la relative indifférence de l'écrivain à l'égard du travail de l'artiste. Non que Barrie soit foncièrement mécontent du travail de Rackham (sinon il n'aurait pas même accepté l'idée du livre illustré), mais l'esprit de l'écrivain est à cette époque probablement ailleurs : l'état d'Arthur Llewelyn Davies est toujours préoccupant - il s'éteindra le 19 avril de l'année suivante ${ }^{14}$. 

« partager sa gloire » avec l'illustrateur. Plus tard, en 1911, il fera illustrer Peter and Wendy par Francis Donkin Bedford, un artiste bien moins connu à l'époque que Rackham. Pour quelle raison? Une hypothèse (parmi d'autres) : l'amour-propre d'écrivain de Barrie voyait en 1911 sans doute d'un œil sinon mauvais, du moins méfiant, l'idée de " partager sa gloire " à nouveau, à un moment où il n'en avait plus véritablement besoin, avec un artiste qui d'une part était lui-même à l'acmé de sa célébrité en Angleterre, et qui d'autre part se permettait d'interpréter les textes à sa manière.

Arthur Rackham imprime en effet sa marque dans l'illustration qu'il fait du texte de Barrie, à commencer par la manière dont il représente le héros de Barrie. Peter Pan est un personnage difficile à représenter dans la mesure où c'est un enfant à l'âge particulièrement indéterminé. Barrie introduit son personnage au début du deuxième chapitre ; après avoir mentionné le fait qu'il était connu aussi bien de la mère que de la grand-mère des enfants de 1900, le narrateur continue ainsi :

Naturellement, cela prouve aussi que Peter a un certain âge, mais comme en réalité il a toujours le même âge, cela n'a aucune espèce d'importance. Son âge est d'une semaine, et quoiqu'il soit né depuis longtemps, il n'a jamais eu d'anniversaire, et n'a pas la moindre chance d'en avoir jamais un. La raison en est qu'à l'âge de sept jours il s'est échappé de l'état d'être humain ${ }^{16}$.

Si l'on suit le texte à la lettre, donc, Peter Pan s'est arrêté de grandir à l'âge d'une semaine, et a depuis cessé de subir le cours du temps («il n'a jamais eu d'anniversaire »). La logique voudrait donc que l'illustrateur représente un nourrisson, et c'est ce que Rackham fait quand il représente Peter Pan s'envolant pour la première fois de chez ses parents (« Away he flew, right over the houses to the Gardens »). Cependant le texte pose par la suite problème à l'illustrateur, dans la mesure où Peter Pan ne tarde pas à pratiquer des activités (il marche, court, discute, navigue dans un bateau, etc.) tout à fait inenvisageables pour un garçon d'une semaine. Nous passons sur les détails, mais la suite des illustrations représente un Peter âgé d'un an et demi environ : les illustrations qui le représentent en train d'essayer de s'échapper de l'île des oiseaux avec un cerf-volant («Peter screamed out, "Do it again!" and with great good-nature they did it several times »; «A hundred flew off with the string, and Peter clung to the tail »; " After this the birds said that they would help him no more in his mad enterprise ») montrent un enfant bien joufflu, tenant sur ses jambes, avec une abondance de cheveux qu'il n'avait pas au départ.

Rackham, dans les illustrations suivantes («Peter Pan is the fairies' orchestra »; «They all tickled him on the shoulder »), ne fera jamais passer le cap des dix-huit mois ou des deux ans au héros de Barrie, alors même que le texte place Peter dans des positions psychologiques et physiques tout à fait invraisemblables pour un bébé de cet âge-là : ainsi de la discussion et du début d'idylle avec Maimie Mannering - personnage qui d'après le texte a quatre ans, et est représentée comme telle par Rackham. Ces passages dans lesquels Peter pratique des activités impossibles pour un bébé de moins de deux ans comme par exemple le fait de discuter avec une petite fille de quatre ans - sont volontairement ignorés de Rackham, ceci pour deux raisons : tout d'abord le rapport entre Peter et Maimie préfigure celui de Peter et de Wendy dans la pièce de théâtre, et tout se passe comme si Rackham tenait absolument à occulter tout lien entre le premier et le second Peter, tout lien possible avec la pièce de théâtre "concurrente ». Ensuite, pour des questions de vraisemblance iconographique relativement étrangères au 
fonctionnement du texte, Rackham tient à conserver une identité visuelle à Peter Pan qui le cantonne au premier âge de l'enfance ${ }^{17}$. Cette identité visuelle marquée explique peutêtre - mais seulement en partie - le fait que l'illustrateur n'ait pas été choisi en 1911 pour mettre en images le Peter Pan beaucoup plus âgé, déjà petit garçon, de Peter and Wendy : il revenait à un autre illustrateur, Francis Donkin Bedford, de donner une identité visuelle différente à ce personnage insaisissable, dont les aventures à Neverland sont à peu près incompatibles avec le Peter Pan imaginé par Rackham. Et si l'identité visuelle donnée par Rackham semble aujourd'hui bien incongrue, c'est du fait du succès généralisé de Peter and Wendy, avec le type inventé par Bedford et réimaginé ensuite par Walt Disney ${ }^{18}$.

Alors même que le Peter Pan de Neverland était tout à fait connu de Rackham à l'époque où il dépeint celui de Kensington - la pièce de théâtre battait alors son plein - Rackham ne tente pas de concilier les deux Peter, mais particularise au contraire celui qui l'intéresse afin de mieux affirmer son interprétation personnelle du texte. Il imprime sa marque au premier pour des raisons de vraisemblance iconographique autant que pour des raisons commerciales : il s'agit de publier un livre où les illustrations fassent œuvre en face du texte, comme le montre enfin la mise en page de l'édition de 1906 où toutes les images sont regroupées à la fin de l'ouvrage. Les illustrations sont certes assorties de légendes tirées du texte et imprimées sur les serpentes, légendes qui relient encore les images au texte illustré. Mais elles prennent place dans un dispositif éditorial qui les présente véritablement comme une seconde œuvre, dotée d'une unité tout aussi inaliénable que celle du texte ${ }^{19}$.

\section{Barrie réenchanté par Rackham}

18 Prenons place maintenant davantage dans le corps du récit afin de voir la manière dont l'artiste a pu mettre celui-ci en images. L'une des particularités de Peter Pan in Kensington Gardens par rapport aux autres aventures racontées dans la pièce de théâtre et le roman de 1911 est que celles-ci s'y déroulent dans une géographie tout à fait réelle, à laquelle est donnée, malgré de nombreuses modifications, une crédibilité très importante par un certain nombre de moyens tant textuels que paratextuels. L'un de ces moyens est la carte donnée en frontispice dès la première édition de The Little White Bird en 1902, et qui est redessinée par Rackham en 1906 sous le contrôle de Barrie - comme on l'a vu dans la correspondance ci-dessus - pour être placée en page de garde. L'autre moyen est la longue description que fait Barrie des jardins de Kensington au cours du premier des six chapitres. Ce chapitre est intitulé "Le Grand Tour des jardins", dans une allusion facétieuse au Grand Tour des jeunes aristocrates anglais qui, depuis le $\mathrm{XvII}^{\mathrm{e}}$ siècle, faisaient le «tour» de l'Europe continentale (avec pour destination privilégiée l'Italie) pour parfaire leur éducation avant leur entrée dans la vie active. Ainsi Barrie invite à découvrir le monde dans les jardins : ce voyage a une portée pédagogique, à l'instar du Grand Tour dont l'objectif était l'apprentissage de la vie, et constitue un véritable parcours d'initiation aux secrets des jardins, monde à part entière.

Ces secrets résident en grande partie dans la vie féerique qui anime les jardins la nuit tombée - et qui continue pendant la journée, quoique de manière plus discrète. Barrie met néanmoins du temps à parler des fées, ne les faisant apparaître qu'à partir du chapitre II avant d'y consacrer les chapitres IV et V de son récit, comme s'il convenait d'abord d'ancrer ce dernier dans la réalité de la vie quotidienne afin de mieux emporter, en un second temps, le lecteur dans un monde merveilleux. 

donc inexistantes. Et pourtant, dès la première illustration, consacrée à la deuxième phrase de l'incipit, "The Kensington Gardens are in London, where the King lives », Rackham encombre son dessin de créatures féeriques. D'une certaine manière, l'artiste annonce ainsi la "couleur", le ton merveilleux d'aventures qui ne sont dans le texte au départ consacrées qu'à la description de divers jeux, mœurs et anecdotes enfantines. L'image, par son décalage d'avec le texte, crée ainsi un effet d'attente : quelles sont ces fées dans l'image dont le texte ne parle pas? En même temps, Rackham ne néglige pas non plus l'ancrage réaliste caractéristique du premier chapitre: la même image représente en arrière-plan le roi Édouard VII, qui est en effet mentionné dans le texte, manière cette fois-ci d'ancrer le récit dans l'ici et le maintenant de l'Angleterre des années 1900 . On assiste donc au paradoxe d'une illustration d'incipit qui place au second plan la lettre du texte et qui annonce à l'inverse au premier plan quelque chose que le texte ne commencera à décrire que bien plus tard : manière sans doute pour Rackham de montrer que l'essentiel des jardins se trouve dans la féerie qu'on y trouve, mais surtout de jouer avec les conventions de l'illustration qui veulent que l'illustrateur suive scrupuleusement la lettre du texte sans y déroger. Tout au contraire, l'illustrateur ici s'amuse à apposer, dès l'ouverture, sa marque personnelle: Rackham est alors connu, depuis son illustration des Contes de Grimm en 1900 et surtout de Rip Van Winkle d'Irving en 1905, pour être le peintre des fées par excellence, et l'arbre anthropomorphe qui abrite sous sa ramure les êtres féeriques fonctionne également comme une marque de fabrique tout à fait reconnaissable ${ }^{20}$. tout au long du récit, de voir des fées là où il n'y en a pas. Ainsi de «The Serpentine is a lovely lake, and there is a drowned forest at the bottom of it [...]», ou encore de « Old Mr. Salford was a crab-apple of an old gentleman who wandered all day in the Gardens ", pour nous cantonner à des exemples illustrant le premier chapitre. Cette dernière illustration nous met sur la voie d'une autre manière encore pour Rackham d'interpréter le texte: dans ce dernier, il n'est nulle part allusion, lors des paragraphes consacrés à M. Salford, de l'Albert Memorial que Rackham représente pourtant en arrière-plan. L'illustrateur glose donc le texte dans le sens de la féerie comme dans celui de l'ancrage réaliste: l'Albert Memorial, comme le roi Édouard VII dans la première illustration, se trouve à l'arrière-plan de ses images de la même manière que le récit a pour toile de fond le Londres du XIX ${ }^{\mathrm{e}}$ siècle $^{21}$.

Rackham, s'il insiste sur la présence de fées et d'arbres anthropomorphes là où le texte n'en requiert pas, insiste donc à part égale sur l'ancrage géographique du récit dans la topographie des jardins. À côté d'images sorties tout droit de l'imagination de l'artiste comme « The fairies have their tiffs with the birds » ou encore « The fairies are exquisite dancers ", dont les arrière-plans laissés vierges contribuent à donner une impression d'irréalité, d'autres compositions insistent au contraire sur des détails de l'architecture ou de l'ameublement des jardins de Kensington: ainsi pour «An afternoon when the Gardens were white with snow " qui laisse voir à l'arrière-plan, derrière le rideau d'arbres, le palais de Kensington, ou de «She ran to St. Govor's Well and hid » qui met en valeur graphiquement les grilles séparant les espaces verts des allées.

23

fait ainsi alterner vues naturalistes et vues fantaisistes, de manière à ce qu'à l'échelle de la série ${ }^{22}$ l'impression de merveilleux qui consiste à laisser croire à l'existence de fées derrière la réalité quotidienne puisse se dégager : l'effet d'émerveillement que 
Barrie s'efforçait de ménager progressivement dans son récit résulte chez Rackham d'un entrelacement constant des deux registres, qui parfois se rejoignent entièrement dans des compositions comme "The Serpentine is a lovely lake, and there is a drowned forest at the bottom of it [...]» ou « Old Mr. Salford [...]». Significativement, l'artiste termine sa série non par une ouverture vers le merveilleux, mais par une vue tout à fait naturaliste des deux bornes que le narrateur s'amuse à prendre pour des pierres tombales («I think that quite the most touching sight in the Gardens is the two tombstones of Walter Stephen Matthews and Phoebe Phelps »). Dans cette image de clôture, pas de fées cachées dans les racines, pas de formes étranges données aux arbres, mais des personnes adultes (et en cela imperméables à la féerie) et de simples chaises métalliques de jardin : Rackham prend bien soin de renvoyer le spectateur à la réalité quotidienne, comme pour l'inviter à aller se promener dans les jardins et à y opérer la conversion du regard à laquelle invitent texte et images - à l'instar du regard éploré des promeneurs qui s'apitoie sans raison apparente (pour celui qui ignore les secrets des jardins bien évidemment) sur ces monuments rappelant les limites entre les paroisses de Westminster Saint Mary et de Paddington.

Le témoignage de Walter Starkie déjà cité peut dès lors être compris de deux manières : soit comme l'anecdote d'un artiste un peu fantaisiste qui raconte des histoires aux enfants pour les amuser, soit de manière plus sérieuse - mais tout aussi ludique : il s'agit simplement alors d'un «jeu sérieux » qu'on appelle l'art - comme un témoignage de la manière dont l'artiste travaillait : en un mot comme un exposé de sa poétique. C'est bien évidemment dans ce sens que nous voulons aller quand nous parlons d'une conversion $d u$ regard à laquelle invitent le texte de Barrie comme les images de Rackham. Cette conversion du regard est à proprement parler un réenchantement du monde : l'écrivain comme l'artiste invitent à aller voir derrière les apparences du monde de la vie quotidienne, positive, moderne, pour y déceler la merveille qui y est cachée, et qui n'est révélée qu'à travers le travail actif de l'imagination ${ }^{23}$. Ainsi du bateau-bâton avec lequel les enfants jouent sur le bassin central :

Le navire le plus charmant qui puisse mouiller dans le bassin est ce qu'on appelle le bateau-bâton, car il ressemble davantage à un bâton avant qu'il ne soit mis à l'eau et que vous ne le teniez par une corde. Alors, tandis que vous faites le tour du bassin en le halant, vous voyez de petits hommes courir sur le pont, et des voiles s'élever par magie et prendre la brise; et pendant les nuits difficiles vous faites halte dans un confortable petit port inconnu des hautains yachts. La nuit passe en un clin d'œil, et, de nouveau, votre navire élancé met le nez au vent, des baleines surgissent, vous glissez au-dessus de cités englouties, vous vous frottez à des pirates et vous jetez l'ancre auprès d'une île aux coraux. Vous restez tout seul pendant que se déroulent toutes ces aventures, car deux enfants ensemble ne peuvent s'aventurer bien loin sur le Bassin Rond; et quoique vous puissiez vous parler à vous-même au cours du voyage, donnant des ordres et les exécutant avec célérité, vous ne savez pas, quand le moment de rentrer est venu, où vous avez été ni ce qui a gonflé vos voiles. Votre trésor est pour ainsi dire tout entier enfermé dans vos cales, et il sera peut-être découvert par un autre petit garçon bien des années plus $\operatorname{tard}^{24}$.

Métaphoriquement, les cales emplies de trésors du galion sont ainsi associées aux trésors enfouis dans l'imagination de l'enfance: de manière très romantique, Barrie associe les deux topoi de l'enfance comme lieu d'expression du génie créateur et de l'imagination comme moteur premier de la création artistique. 

en témoigne Walter Starkie, son but n'est pas simplement de suivre pas à pas le récit, mais plus essentiellement de faire voir ( Il me faisait regarder fixement l'un des arbres majestueux aux troncs massifs qui nous entouraient ») derrière les arbres et les différents objets naturels des jardins la merveille qui y réside. Sa série d'illustrations prend ainsi comme points de départ à la fois le texte de Barrie et la réalité visuelle des jardins, points à partir desquels l'imagination de l'artiste démarque ensuite son propre territoire.

Barrie porte une attention particulière, dans son récit, aux différentes espèces végétales et animales qui font le quotidien des jardins. Rackham prend note de cette précision lexicale, et représente des tulipes là où le texte parle de tulipes («When he heard Peter's voice he popped in alarm behind a tulip»), des chrysanthèmes là où il parle de chrysanthèmes ("A chrysanthemum heard her, and said pointedly,"Hoity-toity, what is this?" »), etc. Mais l'artiste prend ensuite ses aises, et dans des images comme «Butter is got from the roots of old trees ", il part de l'idée d'un vieil arbre suggérée par le texte pour s'éloigner ensuite radicalement de la réalité des jardins de Kensington. Sans avoir vraiment les moyens de le vérifier directement - le parc arboricole des jardins de Kensington ayant probablement vécu de nombreuses altérations depuis les années 1900 on peut néanmoins douter qu'un tel arbre aussi imposant, aux branches tordues et aux racines protubérantes, ait vraiment eu sa place dans le cadre des jardins de Kensington ${ }^{25}$ : à tout le moins l'illustrateur s'inspire-t-il des arbres existants pour s'en éloigner aussitôt, et donner une composition de forêt lugubre en lieu et place du paisible jardin auquel on aurait pu s'attendre. L'artiste s'inspire des formes existantes mais les prolonge dans son imagination pour les faire accoucher de la vie qu'elles recèlent; ainsi les arbres de Kensington sont-ils proprement animés : dans «An elderberry hobbled across the walk, and stood chatting with some young quinces ", où les protubérances du bois dues à la taille des jardiniers peuvent aisément être transformées en tête humaine, l'imagination de l'artiste métamorphosant les branches et accidents de l'écorce en autant d'organes anthropomorphes - cheveux, nez, membres, yeux, etc. L'objectif de Rackham est non seulement de " réécrire », de repeindre la réalité, mais aussi de donner corps à la merveille. Pas de réalité sans merveille, mais pas de merveille sans réalité : l'une ne va pas sans l'autre, c'est ce que reprochera Rackham plus tard à Barrie quand il verra l'importance que prennent les illusoires paysages de Neverland par rapport aux détails bien réels des jardins de Kensington.

28 Arthur Rackham était suffisamment connu à l'époque pour qu'on lui demande de s'exprimer sur son travail. Il est ainsi invité en 1910 à un dîner donné en son honneur au Club des Auteurs, où il produit un discours dont les propos sont rapportés par James Hamilton, autre biographe de Rackham :

La théorie principale de Rackham, cependant, était que l'illustrateur doit être un associé de l'auteur, et non son serviteur, non plus qu'une contribution à vendre plus de livres, comme «une page dorée de couverture». "Toute tentative de le réduire [l'illustrateur] à un simple outil entre les mains de l'auteur ne peut conduire qu'au désastre. L'illustration est tout aussi riche de séductions que la littérature; et la seule relation possible entre les deux n'est que l'association, fondée sur l'équilibre et l'affinité. »

Rackham définit trois objectifs principaux à l'illustration : expliciter ce que l'auteur n'a pas clairement indiqué ; ajouter un nouveau centre d'intérêt à celui de l'auteur (« coopération qui s'est toujours révélée efficace »); et enfin - l'objectif le plus fascinant - donner forme à l'émotion ou la sensation de plaisir suscitée par un passage du texte ${ }^{26}$. 
On fera remarquer que les trois objectifs donnés par Rackham engagent l'illustrateur à une infidélité à la lettre du texte, infidélité qu'il recommande donc comme la seule manière d'illustrer qui vaille vraiment la peine. Ces trois objectifs se rejoignent dans une même poétique : partir de la lettre du texte, s'en éloigner, et retrouver au final son esprit (seule manière de ne pas "trahir " l'intention de l'auteur) - ce pourquoi, d'ailleurs, le travail est amplement facilité si la relation entre auteur et illustrateur se fonde en effet "sur l'équilibre et l'affinité ». L'histoire de l'illustration regorge d'exemples de déséquilibre, où un illustrateur tendrait par exemple à vouloir ramener le succès voire la paternité du texte à lui, comme Cruikshank tenta de le faire avec Oliver Twist de Dickens, ou au contraire d'auteurs empiétant sur le domaine de l'illustrateur en bridant sa créativité, comme Lewis Carroll le fit avec John Tenniel pour Alice's Adventures in Wonderland (rien n'indique d'ailleurs que cela conduisit ici à "un désastre », bien au contraire). Rackham prend note de cette histoire parfois tendue des rapports entre auteur et illustrateur, et exprime sa position qui est celle d'un équilibre, fondé à la fois sur le respect de l'intégrité du texte et sur une prise de distance par rapport à lui : ainsi seulement l'illustrateur peut conserver sa propre autorité, aux deux sens du terme.

Cette distance est facile à ménager quand l'auteur est mort, mais pour Peter Pan in Kensington Gardens ce n'est pas le cas, et il est heureux pour Rackham que Barrie ait respecté la liberté que l'illustrateur a pu prendre avec son texte. On demande généralement de manière implicite à l'illustrateur de mettre en exergue les moments les plus dramatiques, les plus signifiants du point de vue narratif. Quels choix opère Rackham? Représente-t-il des moments forts du récit, comme par exemple le débarquement de Peter dans les jardins de Kensington à partir de son nid de grives et l'accueil belliqueux que lui font alors les fées ? le moment où il retourne voir sa mère et où il la voit endormie, hésitant entre la réveiller pour revenir vers elle ou retourner dans les jardins ? celui où Peter est assis à côté de Maimie sur un tronc d'arbre, et où les deux commencent à nouer une idylle impossible? ou encore celui de leur séparation, à l'heure de la réouverture des jardins?

31 Il est intéressant de remarquer que Rackham, sans qu'il oublie non plus absolument tous les moments forts de l'histoire ${ }^{27}$, s'attarde néanmoins essentiellement sur des détails semblant, à la lecture du texte, absolument inessentiels à la narration : non seulement les paysages, qui ont une utilité narrative dans la mesure où elles fournissent le cadre de l'histoire, et explicitent ainsi " ce que l'auteur n'a pas indiqué », mais bien des lambeaux de phrases, des expressions où l'illustration a vraisemblablement pour unique but de " donner forme à l'émotion ou la sensation de plaisir suscitée par un passage du texte ». Ainsi de "There is almost nothing that has such a keen sense of fun as a fallen leaf", splendide phrase assez difficile à traduire, et à partir de laquelle l'illustrateur imagine des fillettes jouant avec des feuilles mortes, dans une oxymore symbolique qui n'est pas sans rappeler, quoique de manière résolument plus dynamique et joyeuse, le sujet d'Autumn Leaves (1855-1856) de John Everett Millais. Barrie interprète ces fillettes comme étant des fées (voir correspondance ci-dessus, lettre du 18 décembre 1906), alors même qu'à cet endroit (le premier chapitre) les fées sont totalement absentes du texte : indétermination iconographique qui montre bien à quel point l'image entretient un rapport ténu et vague avec le texte. Ici, plus que le texte de Barrie, c'est l'opposition de la vivacité joyeuse de l'enfance et de la mélancolie des feuilles mortes qui constitue, avec l'évocation de l'éphémère mouvement du vent en automne, le véritable sujet de l'illustration. 
Ainsi également d'autres compositions comme "The fairies have their tiffs with the birds » ou "Fairies never say, "We feel happy" : what they say is, "We feel dancey" ", qui prennent pour sujets des fragments de phrases que Rackham se réapproprie en marge du récit - ces deux phrases n'occupant aucune place réelle dans la narration, et ayant valeur d'affirmation générale sur les moeurs des fées. Ou encore, de manière certes moins radicale mais tout aussi significative, des moments du récit complètement anecdotiques ou digressifs, mais dont Rackham apprécie au passage la poésie : «Linkmen running in front carrying winter cherries", "The little people weave their summer curtains from skeleton leaves ", etc.

\section{Conclusion}

Nous avons pu voir que tout, dans l'édition illustrée de Peter Pan in Kensington Gardens publiée en 1906, concourait à ménager une position d'équilibre entre l'écrivain et l'artiste, équilibre favorable au second au vu de la traditionnelle position dominatrice de l'écrivain sur son illustrateur: le genre éditorial du «gift book» dont l'intérêt commercial réside dans l'image, l'organisation interne du livre où la série d'illustrations est rassemblée en tant qu'entité autonome à la fin de l'ouvrage, le fait que Barrie ne s'investisse pas outre mesure dans la publication pour des raisons personnelles autant que professionnelles, etc. On a vu également que Rackham exploite cette position d'égalité pour imposer sa marque personnelle à la publication, construisant une identité visuelle au personnage de Barrie qui est incompatible avec celle du personnage de la pièce de théâtre "concurrente ». On a examiné ensuite la manière dont Rackham s'appropriait le texte en se démarquant des impératifs d'une illustration narrative au profit d'une illustration poétique qui lui est propre, et qui met en exergue des moments anecdotiques ou digressifs du texte plutôt que ses grands moments narratifs, ceci afin de mieux mettre en valeur un entrelacement du réel et du merveilleux participant à une entreprise poétique de réenchantement du monde.

Deux thèses s'offrent alors à nous concernant cette appropriation du texte par l'illustrateur. Nous pouvons d'abord y voir l'indice d'une profonde et radicale trahison du texte par l'artiste, qui, par orgueil, serait venu y apposer sa marque personnelle sans tenir compte de la logique narrative (l'arrivée progressive dans le merveilleux) ni des moments symboliques forts du récit (le moment où Peter Pan revient voir sa mère endormie). Toute une dimension du texte, qui sans nul doute peut être considérée comme sa dimension principale, est en effet occultée par Rackham : à savoir le récit de la mort de l'enfant et de son entrée dans une éternité paradoxale, à la fois non voulue et fortement désirée (la scène des barreaux mis à la fenêtre), avec toutes les conséquences possibles sur les rapports avec la mère, les amours impossibles avec Maimie/Wendy, etc.

Il serait malhonnête en effet d'affirmer que Rackham ne trahit pas, par ses choix iconographiques, l'une des dimensions symboliques les plus essentielles du texte. Mais peut-être la lecture que fait Rackham du texte de Barrie en va-t-elle de même que celle que fait Chateaubriand du cardinal de Retz, telle que l'explicite Michel Charles :

Le lecteur contemporain peut considérer que ce qui est intéressant chez Retz, ou séduisant, ce n'est certainement pas le discours politique ni le récit détaillé des cas de conscience et différents débats qui tourmentent le héros, mais bien plutôt ces éclairs descriptifs ou anecdotiques, ces détails, qui en sont précisément les points lumineux. Le 
lecteur contemporain choisit pour sa lecture une ligne de force secondaire, en quelque sorte. En d'autres termes, il va chercher tout de suite un texte fantôme, le sort de la pénombre; il préfère au texte une de ses doublures. Chateaubriand, par une voie qui lui est propre, prépare le terrain à ce type de lecture ${ }^{28}$.

Rackham, de même, omet le propos principal de Barrie pour mettre en valeur une ligne de force qui, bien que parfaitement présente dans le texte, reste néanmoins marginale par rapport au propos principal: à tel point que cette "ligne de force secondaire " disparaîtra du second épisode des aventures de Peter Pan, l'écrivain se concentrant alors sur son enfant éternel en laissant de côté le « réenchantement du monde » qui avait attiré l'attention de Rackham et constitué son affinité avec le texte. Rackham ne trahit donc pas véritablement le texte, mais, par sa lecture personnelle, met en lumière «ces éclairs descriptifs ou anecdotiques » qui constituent comme une doublure du texte, doublure par la suite abandonnée lors de la rédaction du second épisode. Cet abandon constitue un véritable sujet d'amertume pour Rackham, comme en témoigne son fameux commentaire de l'évolution du personnage de Peter $\operatorname{Pan}^{29}$ : Barrie et lui ne suivent ensuite en effet plus les mêmes voies, le premier approfondissant de manière escapiste la «ligne de force principale » qui aura le succès que l'on sait, et le second continuant dans ses illustrations à creuser son sillon d'enchanteur du réel. La lecture de Rackham a en tout cas pour nous le mérite de constituer non seulement une appropriation mais une illumination: l'illustration témoigne certes de problématiques propres à l'artiste que celui-ci projette sur le texte, mais elle met aussi en lumière - conformément à son étymologie - un « texte fantôme » à l'intérieur du texte de Barrie. Fantôme que l'écrivain aura ensuite bien soin d'ensevelir au tréfonds de sa conscience, comme une solution pour sortir de l'île de Neverland que l'on ne voudrait surtout pas voir.

\section{NOTES}

1. Éditions anglaises : Peter Pan in Kensington Gardens, Peter and Wendy, éd. Peter Hollindale, Oxford University Press, « Oxford World's Classics », 1999 (1991). Éditions françaises : Le Petit Oiseau blanc , trad. Céline-Albin Faivre, Rennes, Terre de brume, 2006 ; Peter Pan, ou le garçon qui ne voulait pas grandir, trad. Franck Thibault, Rennes, Terre de brume, 2004. Le catalogue d'exposition consacré à l'oeuvre de Barrie, My Heart in Company, cat. expo. Beinecke Rare Book and Manuscript Library (Yale University, Connecticut), New Haven, Yale University, 2005, est de même significativement orné en couverture d'une illustration d'Arthur Rackham.

2. Voir notre précédent article, dans le prolongement direct duquel ce présent article a été envisagé, "Arthur Rackham dans les jardins de Kensington », Revue des livres pour enfants, $\mathrm{n}^{\circ} 247$, Paris, BNF / La Joie par les Livres, juin 2009, p. 105-114.

3. Même si l'idée d'un enfant éternellement jeune a émergé bien plus tôt dans l'esprit de l'auteur. Pour plus de détails concernant les liens entre vie et œuvre de Barrie et la genèse du personnage, voir l'ouvrage fondamental d'Andrew Birkin, J. M. Barrie and The Lost Boys, Londres, Macdonald Futura Publishers, 1980 (1979), et de manière plus synthétique Peter Hollindale, «Introduction », in Peter Pan in Kensington Gardens, Peter and Wendy, 1999, op. cit., p. VII-XXVIII ; Donna R. White et C. Anita Tarr, «Introduction », in J. M. Barrie's Peter Pan In and Out of Time, A Children's Classic at 100, 
dir. Donna R. White et C. Anita Tarr, Lanham (Maryland), Toronto, Oxford, The Scarecrow Press, 2006.

4. La totalité des illustrations de cette édition est visible sur le site de la bibliothèque de l'université d'Harvard. Url (dernière consultation le 4 novembre 2011): http:// pds.lib.harvard.edu/pds/view/20245699

5. Nous apportons ici correction à notre précédent article (« Arthur Rackham dans les jardins de Kensington », op. cit., p. 106), qui faisait de Barrie le premier instigateur de la commande : rien ne semble montrer que celui-ci ait vu les aquarelles de Rackham pour Rip Van Winkle, contrairement à ce que moi ou Céline-Albin Faivre («Le péan de Pan », in James Matthew Barrie, Peter Pan dans les Jardins de Kensington, trad. Céline-Albin Faivre, Rennes, Terre de Brume, 2010, p. 10) avons pu noter sur le sujet, ni a fortiori que ce soit lui qui ait eu l'initiative de cette collaboration.

6. James Hamilton, Arthur Rackham, l'enchanteur bien aimé, trad. Christine Mouratoff et MariePaule Page, Quimper, Corentin, 1995 (1990), p. 67.

7. La lettre de Barrie est retranscrite dans Derek Hudson, Arthur Rackham, his Life and Works, Londres, Heinemann, 1960, p. 62.

8. Le texte est néanmoins légèrement remanié entre 1902 et 1906 : voir Peter Pan in Kensington Gardens, Peter and Wendy, 1999, op. cit., p. XXIX-XXX.

9. Cité dans James Hamilton, Arthur Rackham, op. cit., p. 71 (trad. Mouratoff, Page).

10. Walter Starkie, cité dans James Hamilton, Arthur Rackham, op. cit., p. 72 (trad. Mouratoff et Page).

11. Cette citation et les suivantes sont reprises de Derek Hudson, Arthur Rackham, op. cit., p. 62-65.

12. Pour le détail des éditions et rééditions de cet ouvrage du vivant de Rackham, voir Richard Riall, A New Bibliography of Arthur Rackham, Bath, Ross Press, 1994, aux dates 1906, 1910 et 1912.

13. Sur le gift book de cette époque, voir Michael Felmingham, The Illustrated Gift Book, 1880-1930, with a checklist of 2500 titles, Aldershot, Scolar Press, 1988.

14. Le livre est d'ailleurs dédié à la famille Davies, manière plus ou moins adroite pour Barrie de montrer son soutien à la famille en détresse : «To Sylvia and Arthur Llewelyn Davies and their boys (my boys).» Cette dédicace est également la seule marque d'appropriation de l'ouvrage qui permette à Barrie de le faire sien, outre bien sûr le texte qui est de lui et sa présence en page de titre: Barrie ne donne pas ici de préface nouvelle à cette réédition d'un texte déjà publié. Son désintérêt (relatif, encore une fois) pour l'ouvrage est certes en partie dû au souci qu'il se fait alors pour la santé d'Arthur Llewelyn Davies, mais aussi au fait qu'il s'agit davantage d'un ouvrage d'art partant de son texte plutôt que de son texte agrémenté d'illustrations: en bref, l'écrivain n'est pas partie prenante dans cette édition illustrée.

15. Ce qui toutefois n'empêchera pas Arthur Rackham de composer de nouvelles illustrations (une en couleur pour le frontispice, et une quinzaine d'autres en noir en hors-textes et vignettes) en 1912 pour une nouvelle édition (toujours chez Hodder \& Stoughton) de ce texte de Barrie. Ce travail supplémentaire reste toutefois dans la continuité de celui effectué pour l'édition de 1906. Du point de vue de l'éditeur, cette édition augmentée - de même que le portefeuille contenant un choix d'illustrations de Rackham vendues sans le texte, publié la même année - vise très probablement (nous n'en avons néanmoins pas de preuve par les sources) à accompagner l'inauguration de la statue de Peter Pan réalisée la même année par Sir George James Frampton RA pour les jardins de Kensington.

16. " Of course, it also shows that Peter is ever so old, but he is really always the same age, so that does not matter in the least. His age is one week, and though he was born so long ago he has never had a birthday, nor is there the slightest chance of his ever having one. The reason is that he escaped from being a human when he was seven days old ", Peter Pan in Kensington Gardens, op. cit., p. 12. Toutes les traductions du texte de Barrie sont de notre fait. 
17. Il peut paraître peu pertinent que de s'attacher à la question de l'âge de Peter - après tout, en cessant de grandir il n'est pas seulement devenu immortel mais éternel, sa vie s'écoulant en dehors du temps : il serait alors absurde de s'inquiéter de ce qu'il peut faire ou de ce qu'il ne peut pas faire selon son degré de croissance. Seulement, si dans le texte Peter garde une même identité grâce à son nom, le problème de sa croissance et de la forme de son corps passant au second plan, dans l'image seule cette dernière permet de l'identifier visuellement, et le texte de Barrie représente alors un véritable défi pour l'illustrateur s'il veut que le personnage «se tienne ", garde son unité : l'artiste perdrait le lecteur s'il représentait un Peter Pan qui ne cesse de changer de taille alors même que le texte précise que l'une de ses caractéristiques les plus marquantes est qu'il ne grandit pas. Autrement dit: si dans le texte de Barrie Peter est une idée (celle de l'enfance) presque plus qu'un personnage, sa représentation iconographique est problématique.

18. Le type inventé par Bedford est lui-même probablement calqué sur le type androgyne incarné par Maude Adams, qui joue Peter Pan dans la pièce de théâtre, ainsi que sur les photos des enfants Davies réalisées par Barrie. Quant au type inventé par Disney, il doit beaucoup à celui de Bedford, voir Robin Allan, Walt Disney and Europe, Bloomington, Indianapolis, Indiana University Press, p. 217-222.

19. Le passage à la forme du livre illustré à partir de 1912, où les illustrations sont distribuées au long du texte avec de nouvelles illustrations en noir, rend moins aisément perceptible cette cohérence à l'échelle de la série. Significativement, en 1912 sort aussi, toujours chez Hodder \& Stoughton, un portefeuille contenant les reproductions d'une douzaine d'images de Rackham pour le texte de Barrie; l'autonomie artistique des illustrations est ici telle que la série (incomplète toutefois) se vend séparément : elles constituent comme un autre « texte».

20. Dans une autre illustration de la même série, "A chrysanthemum heard her, and said pointedly,"Hoity-toity, what is this?" ", Rackham se représente même sous les traits d'un chrysanthème, autoportrait montrant bien le caractère de «signature » que l'artiste attribue à ses fameux « arbres à la Rackham » : manière encore une fois de s'affirmer plutôt que de s'effacer en tant qu'auteur.

21. Barrie situe en effet en partie dans le $x x^{\mathrm{e}}$ siècle les aventures soi-disant «immémoriales » de Peter Pan quand il introduit une anecdote concernant le poète Shelley au début du chapitre III. Transformant ainsi le mythe en légende.

22. Série qui, rappelons-le, a dans l'édition de 1906 une existence indépendante de celle du texte dans la mesure où toutes les illustrations sont réunies en fin d'ouvrage.

23. Notre point de vue diffère ici sensiblement de celui de Céline-Albin Faivre pour qui «les Jardins - ceux de Barrie, en tout cas - ne sont pas plus réels que Never Never Never Land, ils paraissent simplement plus aisés à imaginer et à habiter » ("Préface - Le péan de Pan », op. cit., p.11). Si les jardins de Kensington participaient de la même irréalité que Neverland, le fonctionnement narratif serait autre: Barrie ne jouerait pas avec le lecteur pendant tout le premier chapitre à travestir une réalité censément familière. Il ne s'agit pas ici d'habiter dans les jardins (personne n'y habite d'ailleurs, si ce n'est Peter et les fées), ni même de les imaginer, mais bien de les voir, justement pour que puisse s'opérer la conversion du regard (l'« enchantement ») qui fait advenir la merveille à la lisière de la réalité quotidienne : modalité du merveilleux bien différente de celle à l'oeuvre dans Peter and Wendy.

24. «For the sweetest craft that slips her moorings in the Round Pond is what is called a stickboat, because she is rather like a stick until she is in the water and you are holding the string. Then as you walk round, pulling her, you see little men running about her deck, and sails rise magically and catch the breeze, and you put in on dirty nights at snug harbours which are unknown to the lordly yachts. Night passes in a twink, and again your rakish craft noses for the wind, whales spout, you glide over buried cities, and have brushes with pirates and cast anchor on coral isles. You are a solitary boy while all this is taking place, for two boys together cannot 
adventure far upon the Round Pond, and though you may talk to yourself throughout the voyage, giving orders and executing them with dispatch, you know not, when it it is time to go home, where you have been or what swelled your sails; your treasure-trove is all locked away in your hold, so to speak, which will be opened, perhaps, by another little boy many years afterward. ", Peter Pan in Kensington Gardens, op. cit., p. 7-8.

25. Et ce même si une partie des jardins était d'aspect plus «sauvage " naguère qu'aujourd'hui, voir Lisa Chaney, Hide-and-Seek with Angels, op. cit., p. 124.

26. James Hamilton, Arthur Rackham, op. cit., p. 91.

27. Ainsi de « Away he flew, right over the houses to the Gardens »; « Put his strange case before old Solomon Caw "; "He passed under the bridge and came within full sight of the delectable Gardens ", etc., ou encore de passages moins importants narrativement mais mettant en scène des événements ou des personnages emblématiques, comme "Peter Pan is the fairies' orchestra ", ou encore "Queen Mab, who rules in the Gardens ».

28. Michel Charles, Introduction à l'étude des textes (Seuil, 1995), cité dans Sophie Rabau, L'Intertextualité, Paris, Flammarion, 2002, p. 223.

29. «Peter Pan aurait pu devenir le même personnage traditionnel [que Rip], si son auteur n'avait pas créé deux Peters entièrement différents, celui de Kensington étant un personnage de chair et d'os, et l'autre Peter le symbole d'une éternelle enfance. J'ai toujours pensé que c'était bien dommage. La pièce, évidemment, a entièrement éclipsé le livre, par le pouvoir d'attraction propre au spectacle, mais je regrette qu'on ait laissé échapper l'occasion de peupler éternellement les jardins de Kensington, comme le livre aurait pu le faire. Je pense que les terres de Never-Never sont de pauvres et maigres ersatz de celles de Kaatskills [lieu où se déroule l'action de Rip van Winkle] et de Kensington, avec leur extraordinaire ouverture sur l'imaginaire. Regardez la puissance du mythe attaché à un lieu. Le Rhin. Les montagnes de l'Atlas. L'Olympe. Le Brocken. Dick Whittington. Le Roi Arthur. » Lettre d'Arthur Rackham à Eleanore Farjeon, décembre 1913, cité dans James Hamilton, Arthur Rackham, op. cit., p. 72-74 (trad. Mouratoff et Page).

\section{RÉSUMÉS}

Le premier illustrateur du personnage de Peter Pan est aussi paradoxalement celui qui s'éloigne le plus, tant du point de vue iconographique que symbolique, du mythe que celui-ci deviendra. Que ce soit dans le cadre économique du marché du livre illustré ou dans celui de l'interprétation $\mathrm{du}$ texte, Arthur Rackham, en effet, opère une véritable appropriation du premier épisode racontant les histoires de Peter Pan, Peter Pan in Kensington Gardens, qui sera largement éclipsé par le second racontant les aventures de Peter dans le pays de Neverland. S'éloignant parfois du texte et des conventions de l'illustration narrative, l'illustrateur en poétise le contenu et en extrait des fils qui, bien que présents dans le texte, n'en constituent pas la trame principale. Ce faisant, les images de Rackham constituent une lecture du mythe de Peter Pan qui sera contrariée par l'évolution du personnage dans l'œuvre de Barrie, mais qui n'en éclairent pas moins une facette essentielle.

The first illustrator of Peter Pan has also been, paradoxically, the one who has taken the greatest liberties with the character, in relation to the myth he will become, both iconographically and symbolically. Arthur Rackham has truly appropriated for himself the first episode of Peter Pan's adventures, Peter Pan in Kensington Gardens, which will be followed by the much better known 
adventure in Neverland. This appropriation works both in the economic context of the market of book illustration, and for what concerns the artist's interpretation of the text. The illustrator enhances the poetic value of the story. He focuses on secondary narrative strands, taking some distance from the text itself and from the conventions of literary illustration. Because of that, Rackham's images constitute an interpretation of Peter Pan's myth that will be contradicted by the further evolution of the character, but which is still useful to shed light on some important aspects of it.

\section{AUTEUR}

\section{FRANÇOIS FIÈVRE}

Docteur en histoire de l'art, chercheur associé à l'INTRU (Université François Rabelais, Tours) 